\title{
Diferencias Legislativas para implementar criterios globales sobre la Responsabilidad Social Empresarial
}

\author{
Leidy Karina Pérez Trejos \\ Corporación Universitaria de Ciencia y Desarrollo - UNICIENCIA, \\ Bucaramanga, Colombia \\ Universidad de Los Andes, Programa de Doctorado en Ciencias Contables, \\ Mérida, Venezuela \\ Correo electrónico autor: leidyk.perez@unicienciabga.edu.co \\ Recepción: 27 Septiembre 2017 \\ Aprobación: 27 Noviembre 2017
}

\begin{abstract}
Resumen
Este articulo busca conocer las diferencias legislativas que se encuentran en la implementación de criterios globales de responsabilidad social empresarial, y la afectación de estas diferencias en las organizaciones y en el mercado global. En una primera instancia se trata la esencia de la Responsabilidad Social Empresarial (voluntariedad) y cómo influyen en ella las diferentes perspectivas o puntos de vista como es entendida la Responsabilidad Social Empresarial. Actualmente, continúa siendo un actor importante en el ámbito empresarial, dado que a la diversidad de pensamiento y perspectiva que existen frente al tema, generando diferencias legislativas locales, regionales, nacionales e internacionales, haciendo necesario una fundamentación sobre la voluntariedad desde una vista del derecho.
\end{abstract}

Palabras clave: responsabilidad social, legislación, voluntariedad, sostenibilidad, protonormas. 


\title{
Legislative differences to implement global criteria on Corporate Social Responsibility
}

\begin{abstract}
This article seeks to know about the legislative differences that are found in the implementation of global criteria of corporate social responsibility, and the impact of these differences in organizations and in the global market. In the first instance, the essence of Corporate Social Responsibility (voluntariness) is discussed and how different perspectives or points of view influence it, as Corporate Social Responsibility is understood. Currently, it continues to be an important actor in the business field, given the diversity of thinking and perspective that exists in the subject, generating local, regional, national and international legislative differences, making necessary a foundation on the voluntariness from a view of law.
\end{abstract}

Keywords: social responsibility, legislation, voluntariness, sustainability, protonormas.

\section{Introducción}

Actualmente la Responsabilidad Social se encuentra presente en cada una de las acciones de la sociedad y por ende de las organizaciones, llamando la atención en ella, el aspecto normativo o jurídico en el cual se soporta, para dar aplicabilidad a los objetivos delineados por los organismos encargados de emitir este tipo de documentos, y que han de ser la directriz en su implementación. Para ello, se ha utilizado una revisión bibliográfica que permita realizar una descripción de las diferencias legislativas globales y las nacionales a la hora de realizar una adopción o adaptación de la normativa.

En el desarrollo de este trabajo, es claro que la RSE presenta una falta de fundamentación jurídica que ayude a solidificar y dejar en claro el concepto de RSE, y hacer conciencia de que esta debe ser tomada con carácter voluntario y no con carácter de beneficio económico adquirido, por las acciones prestadas.

\section{La Responsabilidad Social Empresarial una mirada desde el campo jurídico}

La Responsabilidad Social Empresarial (RSE), a pesar de ser un fenómeno voluntario que busca conciliar el crecimiento y la competitividad, integrando al mismo tiempo el compromiso con el desarrollo social y la mejora del medio ambiente (Domínguez, 2008), trasciende la voluntariedad de las mismas empresas y organizaciones económicas y productivas, y no hay país, hoy en el mundo, donde la RSE, no sea regulada por el Estado, y le intente dar un estatus jurídico.

En lo precedente, hay una preocupación 
global porque la conciliación de empresa y producción con lo social como responsabilidad, con Rugeles (2012), opere

...la perspectiva teleológica del Pacto Global de promover el diálogo social para la constitución de una ciudadanía corporativa global, que posibilite conciliar intereses de empresas, con demandas y valores de la sociedad civil, los proyectos de la ONU, sindicatos y Organizaciones No Gubernamentales (OGNs), sobre la base de 10 principios que transversalizan el conjunto de actividades que materializan las empresas y que están relacionados con los valores fundamentales en materia de: derechos Humanos, normas laborales, medio ambiente y lucha contra la corrupción (p.68).

Relevante, entonces reconocer que existe un interés no sólo privado, sino también público, porque haya un ente único que realice las disposiciones legales, en esos términos, en que opera una legislación internacional en materia penal, civil, que también se encargue de hacer que la responsabilidad social empresarial, opere en la globalidad.

Más cuando con Rojas (2011) los mercados dejaron con la apertura económica por el suelo, la economía de mercados locales y nacionales. Luego se infiere la RSE, es de compromiso también del mercado global.

El fenómeno de la RSE, por lo tanto debe observarse más allá de la perspectiva de los años 60, que lo asumía como filosófica "sino que es parte de la gestión empresarial en la década de 70, por consiguiente en los 80's lleva a la conformación de un contexto socialmente responsable y a la dirección estratégica por medio de la teoría de los stakeholders o grupos de interés que rodean a la organización en su devenir comercial del nuevo milenio (Vargas, 2013, p. 47)”.

La RSE ha dejado de ser un evento meramente especulativo y de pensamiento, y ha entrado en el terreno de lo pragmático. Se le mira desde su operatividad, para que entre a satisfacer esos logros de inclusión social, de desarrollo humano, de unos derechos humanos que no sean letra muerta, y una protección ambiental, rodeada de políticas económicas sostenibles.

El problema sobre la operatividad global de la RSE, se presenta en que hay diversas miradas de observar la responsabilidad social de la empresa. Aún persisten miradas locales, nacionales, regionales, continentales en su observancia, y se hace necesario implementar estrategias que conduzcan a lograr esa afinidad, que permita poner la RSE, en un contexto más global.

Ante este problema surge el interrogante 
de cómo sortear las diferencias legislativas ante la necesidad de fijar criterios globales de RSE, $\mathrm{y}$ es que esas diferencias legislativas son las que no permiten que haya unidad de criterios para poner a operar de manera global la RSE, viéndose por lo tanto afectadas las comunidades para lograr que el desarrollo, la productividad y la competencia, no las excluya del beneficio de la participación de la riqueza.

Como lo manifiesta Gualteros (2012), la RSE, ha buscado conciliar economía con responsabilidad social, pero si la normatividad es varia y dispersa, o presenta diferencias de carácter legislativo, las empresas ante la falta de claridad, pueden fijar la RSE, como les convenga, y perderse el espíritu de esta figura.

Por eso, es necesario que la iniciativa legislativa tienda a su armonización, que no es otra cosa que ponerse de acuerdo sobre lo esencial, para que con Gualteros (2012), haya una legislación única pensada en lo fundamental, de tal manera que ninguna comunidad, quede por fuera de los beneficios de la RSE. Pues en últimas lo que se busca es inclusión y no exclusión.

Naturalmente hay dificultades para sortear la uniformidad legislativa, que en última es una, y los acuerdos, pactos y convenios globales otra, por su no obligatoriedad jurídica, como también por otra parte, los códigos y compromisos internos de las empresas situadas en el mercado global, pues en la postura de Gamero (2011): es relevante que surtan coincidencias entre legislaciones internas sobre el manejo de RSE, especialmente en los países en vías de desarrollo y latinoamericanos; ojalá por bloques regionales, opere un marco jurídico con más aristas de afinidad que de diferencias, pues los mercados globales fuerzan a ello, y a que las empresas en sus códigos y compromisos internos sobre la responsabilidad social empresarial, lo flexibilicen para que no entren en choque con la legislación interna.

En lo precedente, la uniformidad legislativa debe sortear problemas que conlleven a alcanzar un logro que vaya más allá de la armonización legal interna, pues esta no se logra mientras no se defina el espíritu jurídico que debe reglar los presupuestos y fundamentos de la RSE, frente a la cual se dan dos enfoques: uno el de la voluntariedad, que para sus críticos soslaya el derecho (Vargas,2012), y el otro el de la RSE, observada como derecho, por ser objeto de la producción, y esta genera derechos (Ermida, 2004).

Leidy Karina Pérez Trejos 
Por eso, la importancia de conocer la naturaleza de estos dos enfoques si se buscan generar estrategias, para salvar las dificultades de la diferenciación legislativa, y alcanzar la uniformidad jurídica, pues el problema que convoca este ensayo es de carácter legal, y en él tienen que ver los cuerpos legislativos. Y más cuando estos modelos enfrentan en la concepción de la RSE.

Para Ermida Urriarte, Gianibelli \& Orsatti (2009) se ha realizado una fuerte crítica con respecto responsabilidad social entendida por los entes económicos, puesto que, aun no se define su voluntariedad ni exigibilidad por parte de terceros, es necesario sumar a esta situación que no se cuenta con una clara redacción en documentos que soportan la RS dificultando su aplicabilidad, puesto que los códigos, normativas y demás legislación son elaborados de arriba hacia abajo, y para algunos actores esto debería ser viceversa.

Dicho lo anterior, se hace necesario ahondar y mejorar el aspecto legislativo de la RSE, donde se muestre como un proceso abierto y que involucre a los verdaderos interesados en el tema, puesto que como lo expresa Pedreros (2009) son derechos fundamentales los que obran en la RSE.

Pero, cuando se pide que se tenga a bien tener en cuenta un amplio espectro de población para determinar los ámbitos jurídicos de la legislación de la RSE, lo que se busca es que ésta se asuma con un amplio y vasto jaez en su construcción. Es indudable, que es necesario proponer esta mirada, pues una mirada amplia, dual, es más efectiva en la construcción de saber y ciencia, que la unilateral que es parcial (Méndez, 2010).

Por eso no hay que desechar lo que se pueda tomar de ambos enfoques: el de la voluntariedad y el jurídico en estricto. Se observa, por algunos que la voluntariedad entra en el juego de lo no vinculante, por ser protonorma, esto que llaman soft law, y que Merino (2006), citado por (Ermida Urriarte, Gianibelli, \& Orsatti, 2009) desmenuza para lograr una tesis interesante, en los propósitos de este trabajo. Las protonormas son explicadas como aquella antelación, sugerencia o propósito cuya finalidad no es imponer lo escrito o propuesto, por el contrario, son recomendaciones de lo que podría ayudar a mejorar procesos $\mathrm{u}$ actividades que degeneran en consecuencias no deseadas.

Es necesario aclarar, que este tipo de 
documentos presentan algunas consideraciones como son: la falta de divulgación y conocimiento por parte de las naciones en el caso de los países Latinoamericanos, una segunda apreciación es el optimismo que impulsa a la conformación de gremios en defensa de este tipo de proyectos, logrando en algunos casos la adopción de los mismos, adquiriendo el carácter normativo, por ende, se convierten en de obligatorio cumplimiento.

Como se observa, la voluntariedad, afincada en el soft law, puede desembocar en norma, en evento de ley, por lo que una estrategia para salvar el problema de la legislación diversa, es acercar lo jurídico y la protonorma, en lo que vincula, para que se hagan corpus jurídico.

Bien se defiende el acercamiento de la protonorma para juridicarla, cuando (Ermida Urriarte, Gianibelli, \& Orsatti, 2009), reconoce que

Si las protonormas, códigos y subnormas de RSE no constituyen normas jurídicas propiamente dichas, si más bien se trata de normas éticas o propósitos, entonces no se puede hablar de una eficacia jurídica en el sentido de la posibilidad de alcanzar su aplicación forzosa o una reparación por el incumplimiento. Sin embargo, ya se dijo que no deben minimizarse los efectos que estos códigos voluntarios podrían llegar a tener, sea como impulsores de normas futuras o como inductores de determinadas conductas. Y corresponde agregar aquí que también pueden ser relevantes los mecanismos de evaluación, seguimiento o aplicación eventualmente previstos en los propios códigos de conducta, aunque en general, cuando existen, no están suficientemente desarrollados (p.17).

Las protonormas, o el afán de la voluntariedad en la construcción de la RSE, no deben soslayarse ipso facto. Porque son en esas para normas, donde a futuro puede materializarse una mejor construcción y aplicabilidad legal de la Responsabilidad social, con impacto, y no sean letra muerta; pues no hay que olvidarse que la RSE, nace como legalidad más de la voluntad, que del mismo ámbito jurídico, al observarse un derecho que surge por impacto de lo productivo (Valero, 2008).

\section{Y, en lo procedente es oportuno reconocer}

que la conciliación de la voluntariedad y lo jurídico, en ese afán de superar las diferencias legislativas, es una buena estrategia, si se entiende que conducta y legislación son importantes uniformadas, en estos tiempos de mercados globales, pues llegados a este punto, en el caso de las empresas multinacionales, con Ermida (2009), “irrumpe la ardua cuestión de determinar la ley aplicable y la jurisdicción competente, según la cual y ante la cual accionar para obtener la ejecución de una cláusula del código de 
conducta" (p. 18).

Por lo tanto, se demuestra en la aportación de Grau, una vez más, la necesidad estratégica de afinar cuerpo jurídico y voluntariedad, para lograr ese afán de una legislación que supere las diferencias de enfoque, y abra campo a una legislación que interprete el problema, no sólo de una economía global y productividad ídem, sino igualmente de su repercusión social, de desarrollo, ambiental y humana.

En este mismo curso del ensayo, el mercado global, fuerza -así resulte desobligante el término- a manejar globalmente una legislación más universal en materia de regulación de la RSE, por eso no extraña que se hable de una regularización internacional de la RSE.

\section{Para Ermida (2009)}

El contexto de la globalización, las dificultades de los ordenamientos jurídicos nacionales para regularla y la circunstancia de que las principales protagonistas de los códigos de conducta sean las empresas multinacionales, traen naturalmente al debate la posibilidad de proceder a una reglamentación internacional. (p. 20).

En Colombia como se observa lamentablemente, no se ha buscado crear un ambiente legislativo que tienda hacia la armonización legislativa, para lograr salvar ese valladar de las diferencias legislativas.

La pregunta que surge frente la Ley 70 de 2010, es que se trata de una ley que se dispersa en un caleidoscopio de tópicos para legislar, que se relacionan con la responsabilidad empresarial, algunos de raizalidad, pero otros (los más) de carácter tangencial y adventicio (Granados, 2013), por lo que lo fundamentales, lo de arraigo, muchas veces se queda por fuera, por atender minucias, que deberían ser tema de una ley diferente (Vargas, 2013).

La mejor estrategia para abordar las diferencias legislativas, están dadas en los países de Sudamérica, donde obran intereses locales, municipales, nacionales y regionales, sea aquella de la revisión jurídica sobre la RSE, que pueda existir, como proyecto de ley, o como legislación existente.

Es la única manera de saber cuán enfrentadas están las posiciones jurídicamente sobre la manera de ver la RSE. No hay que olvidar, que hasta las mismas leyes, ofrecen dispersión, se divorcian unas de otras, no son trasparentes, frente a un mismo tema.

$\mathrm{Y}$, en últimas lo que se busca es la armonización, pues se reitera los beneficios por 
ser el mercado global, deben ser más integrales, comunitarios y ciudadanos. De ahí la importancia de la revisión como estrategia de apertura de lo legislado en el proyecto; y la concertación en otra fase, de los interesados, en alcanzar una RSE inclusiva: empresarios, trabajadores (a través de sus sindicatos y organizaciones que los representen), cuerpos políticos; integración de grupos de trabajo sobre los tópicos fundamentales, y redacción, finalmente del cuerpo de ley. (Cancelado, 2013)

Sin embargo, sorteadas las dificultades que allanen las diferencias legislativas internas, es necesario romper las que Granados (2012) asume como instancias protolegislativas, y que son hoy definitivas para una RSE de espectro global: la sistematización de espacio regional.

Es sobre este fenómeno de concertación global donde mayormente se debe fijar la atención porque lo que se busca es que la RSE sea de implicación global, y para ello se deben buscar fórmulas de acercamiento y trabajo en el ámbito universal.

Pero no se puede llegar de manera improvisada a sentarse a manteles y decir se va a sistematizar la regulación de la RSE en el mundo, sin mediaciones. Como lo expresa Jaspers (2007) una regulación internacional o global de la RSE, es un proceso que vincula iniciativas oficiales y privadas como comunitarias.

En esto de la sistematización o regularización de RSE, no se pueden dejar por fuera ni las iniciativas privadas o públicas. El Estado y sus gobiernos, avalan los acuerdos, convenios globales, luego debe haber expectativas afinadas respecto de la RSE.

De ahí, siguiendo a Ermida (2009) para que no naufrague un proceso de regularización internacional de la RSE, una primera sistematización, y tal vez la de más amplio espectro, es la que debe tener en cuenta el ámbito geográfico de la reglamentación existente o propuesta. (p. 25)

En otras palabras, se busca reconocer el alcance que tiene la RSE en regulaciones convenidas por un país, en su condición de Estado, haciendo necesario el conocer la estructura de la normatividad que ayuden a la implementación de nuevas leyes o mejora de las existentes, estos acuerdos son en un marco global compuesto por los diferentes sectores de negocio.

Para (Ermida Urriarte, Gianibelli, \& Orsatti, 2009) 
Las normas con pretensión de alcance mundial podrían inscribirse las normas ISO, aunque su carácter privado vuelve muy discutible su inclusión junto con normas de derecho internacional público y con productos; esa mixturación de lo económico y legal (p. 23).

Ante lo expuesto, queda claro que la RSE, no es un tema de carácter local, ni tampoco de importancia de un sector específico de la sociedad. Por lo que, es necesario establecer estrategias de sistematización para regular la RSE, y esta se soslaye del interés neoliberal, afinándose a la inclusión, y a que el desarrollo genere procesos de bienestar para las mismas comunidades y ciudadanos, al hacerlos partícipes de un desarrollo por igual humano.

Es por lo anterior que es necesario tener en cuenta estrategias que apliquen al ordenamiento jurídico moderno, pero que no desechen lo humano, que se expresa en esa vieja idea del Estado-nación (Vásquez, 2011); que no es concepción diferente, a que se viene sosteniendo en este ensayo de la voluntariedad y lo legal.

$\mathrm{Y}$ cuando se propone para que la RSE tenga implicaciones globales, afianzar lo legal con los intereses humanos, no es otra cosa que buscar la conveniencia normativa de una RSE global, pues se busca que sea entendida y atendida por los países firmantes en conclave mundial, por eso debe ser desaprendida de lo enteramente jurídico y construirse, en (Vásquez, 2011) en las expectativas de que su transformación opere.

$$
\text { Indudablemente, las normas deben }
$$
responder a una tipología de regulación, es decir de acuerdo a la capacidad que tengan para echar a andar la RSE. Estas pueden responder a regulación tradicional o directa, sobre derechos exigibles subjetivos donde entran convenios, normas internacionales, pactos, cartas; y a regulación alternativa o indirecta, como técnicas convencionales (Vásquez, 2011).

Regular globalmente, se desprende de este ensayo, no es una tarea sencilla y fácil. Por el contrario es una labor compleja, porque conlleva el implementar procesos, precisamente para sortear las dificultades de las diferencias de legislaciones, y hay que proponer la uniformidad para alcanzar la capacidad de que pueda obrar en un contexto de impacto abierto o global.

Se concluye en este ensayo, que la responsabilidad social empresarial, es un evento que aún está ciernes. Cada país busca legislar, más por novedad, pero sin entrar a considerar si la legislación que propone, o el tipo de regulación internacional que acepta bajo la firma de acuerdos, convenios, cartas, realmente ampara 
a la comunidad o ciudadanía en el impacto global de la productividad.

Pero uno de los problemas más severos, por sortear para alcanzar la globalidad de la RSE, es la de legislar por apariencia, lo que implica que los países que firman estos acuerdos globales, sólo lo hacen por no ser mal vistos por la comunidad internacional.

Por eso la importancia de que los proyectos de ley sobre la RSE, no sólo contengan la juricidad, sino para normas que son las que derivan en la sistematización posterior de la regulación global de la RSE.

Se advierte, que la RSE, no es un evento sin carnadura, que ha surgido por satisfacer un capricho. No. Es el producto de la violación de derechos humanos. Por impactos de la productividad y la economía sobre la ciudadanía y las comunidades, cuando esta las lesiona, es que surge la RSE, o la productividad y la economía generan diferencias sociales, la RPS surge para hacer inclusión social.

La acción global de la RSE, se reitera, vuelve a dejar dudas sobre su efectividad global, y se infiere que son esas carencias de los países en el conocimiento de estrategias, por una parte, para construir un lenguaje que proponga intereses comunes de lo público, lo privado y lo comunal.

Por lo tanto, ese lenguaje para jurídico que debe surgir para que se sorteen las dificultades de unas diferencias legislativas, se debe empezar a construir, convocando a las partes interesadas, en que la RSE funcione a partir de un marco legal de acercamiento. No de otra manera, se pueden alcanzar logros relevantes, y que las comunidades y los ciudadanos del mundo entiendan, que la RSE no es un cuento chino, sino un derecho, que el ejercicio ciudadano puede poner a andar, partiendo de lo nacional hacia lo universal, en su construcción global de legalidad y derecho.

Y qué mejor para darle cierre a este ensayo, que traer a colación, el ejercicio investigativo "Hacia una armonización legislativa en materia de responsabilidad social" como proyecto que en la República de Argentina busca superar las diferencias legislativas, para construir un marco jurídico legal más integral, que dice

El presente trabajo tiene por objetivo contribuir al conocimiento del marco actual legislativo representado por proyectos de ley en materia de RS, en estado parlamentario en la República Argentina, con el ánimo de comprobar la posible armonización normativa entre provincias y a nivel nacional. El cambio de paradigma que vive actualmente el mundo globalizado, hacia la asunción de un compromiso responsable por parte de todas 
las organizaciones en pos de un crecimiento sostenible, requiere un marco normativo legal sobre RS que regule el accionar de todos y cada uno de los partícipes de este movimiento cultural. Este ordenamiento jurídico que viene teniendo lugar en el mundo, comienza a gestarse en Argentina, con la irrupción de numerosos proyectos de leyes tanto provinciales como nacionales (Clide, Coppa, Berry, Magioglo, 2013, p. 13).

Llegados a este punto, se hace necesario fomentar una cultura que brinde un cambio de pensamiento, frente al lenguaje jurídico y de voluntad, buscando dejar a un lado la depredación económica en la que se encuentra sumergida la sociedad, lo que ha deteriorado la salud y el bienestar de todas las comunidades.

Por eso, la importancia de que en las instituciones educativas, desde los años básicos y secundarios, como en las instituciones de educación superior, en las mismas comunidades cívicas, artísticas y culturales, se empodere a la ciudadanía, para el mayor de los ejercicios políticos en un mundo donde la competencia y el desarrollo global, fuerzan a hacer de la gobernanza, la toma de decisiones y el voto, la herramienta para el cambio de paradigma expoliador, por el del desarrollo sostenible y humano.

Realmente, es el empoderamiento de fundamentación sobre la voluntariedad desde

las comunidades, para hacer mejor el mundo, capaz de sostener a todos, y ellos sólo es posible, haciendo entender que es necesario regular la productividad, proponerla desde la generación de energía verde, no contaminante.

Pues no de otra manera se concibe un mundo más amable, y con menor riesgo a su crisis definitiva. El hombre mismo debe imponerse normas que reglen su comportamiento humano, y lo hagan responsable socialmente. He ahí, que estas deben obedecer a un ordenamiento global, porque las consecuencias de la productividad y los efectos de una economía depredadora son de impacto global.

\section{Conclusiones}

Actualmente la Responsabilidad Social continúa siendo un actor importante en el ámbito empresarial, dado que a la diversidad de pensamiento y perspectiva que existen frente al tema, ha generado diferencias legislativas locales, regionales, nacionales e internacionales, y que han conducido a la falta de unidad de criterios que consolide la implementación y manejo de una RSE de carácter global.

Dicho lo anterior se hace necesario una REVISTA DE INVESTIGACIÓN SIGMA / Vol. 04, Nº 1, 2017 (pág. 53-64) 
una vista del derecho, aun cuando los organismos reguladores o emisores de certificación en RSE dicen ser de carácter voluntario, el mercado los establece como un requisito al momento de realizar una contratación, y en cómo se relacionan las organizaciones, cambiando el carácter o esencia de la RSE (la convierte de obligatorio cumplimiento) y excluyente en el mercado actual.

\section{Referencias bibliográficas}

Ackerman, M. (2006). La responsabilidad legal de las empresas transnacionales. Revista de Derecho Social Latinoamérica $n .{ }^{\circ} 1$, Buenos Aires

Arellano, R. (2008). Autorregularse o ser regulado. Diario El Comercio, Lima, Perú, 25 de abril de 2008.

Baylos, A. (2006). La responsabilidad legal de las empresas transnacionales. Revista de Derecho Social Latinoamérica N. ${ }^{\circ} 1$, Buenos Aires

Cancelado, T. (2013). Construcción de un tejido jurídico hacia la armonización global de la RSE.

Clide. (2013). Hacia una armonización legislativa. Buenos Aires

De Sousa, F. (2005). Discurso sobre mercados y responsabilidad social.

Domínguez, M. (2008). Estructura global de EPS. Fondos de Cultura Económica, México.

Ermida, O. (2002). Derechos laborales y comercio internacional. Derecho Laboral, $N .{ }^{\circ} 208$, t. $x l v$, Montevideo.

Grau. (2006). Códigos de conducta global.

\section{México.: Teide}

Granados, J. (2012). Fenómeno sobre juricidad y legalidad social: impactos productivos. México. Fondo de cultura económica

Gualteros, M. (2012). Mercado global y empresa. Bogotá: Norma-Mondadori

Guerrero. (2013). La responsabilidad social empresarial en Colombia. Bogotá. UIS

Hermida, R. (2002). Mercados y globalidad. Barcelona: Tecnos

Jaspers. (2007). (Universidad de Utrecht) en el coloquio De l'irresponsabilité à la responsabilité des entreprises transnacionales dans la globalisation de l'économie, proyecto ester, Université Montesquieu (Bordeaux IV), 28/3/2007

López, R. (2009). Juricidad y protonorma. Bogotá: BOletín jurídico, No. 20

Merino, A. (2006). Soft law y globalización. Estudios de teoría crítica de derecho del trabajo (inspirados en Moisés Meik), Buenos Aires (2006). Mecanismos de regulación de la responsabilidad social de las empresas europeas. La negociación colectiva europea, Manuales de Formación Continuada n. ${ }^{\circ}$ 36, Madrid, Consejo General del Poder Judicial.

Rojas, M. (2011). Categorizaciones de las regulaciones del mercado a partir de la responsabilidad Social. En: Orcus.

Rúgeles, M. (2012). Pacto global y sistematizaciones de la RSE. Barcelona, Morata.

Vargas, R. (2013). Para una filosofía de la RSE. Madrid: Teide.

Vásquez, J. (2011). Tecnicismos jurídicos. México: EFE 